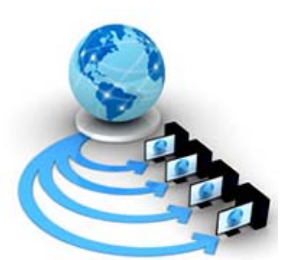

\title{
A SURVEY ON VARIOUS DETECTION TECHNIQUES ON RUN TIME MACHINES
}

\author{
Lavanya $\mathrm{N}$ \\ Computer Science and Engineering \\ New Horizon College of Engineering \\ Bangalore, India
}

\author{
Deepika N \\ Computer Science and Engineering \\ New Horizon College of Engineering \\ Bangalore, India
}

\begin{abstract}
Intrusion Detection Systems (IDSs) are used to identify and report unauthorized or suspicious computer or network activities. Hostbased IDSs, the attention of this paper, are intended to monitor the host system actions, while network-based IDSs monitor network traffic for multiple hosts. Agreeing to their detection techniques, IDSs can also be classified into misuse detection or anomaly detection conditional to whether the intrusion patterns are recognized or not during the design phase.
\end{abstract}

Keywords: Intrusion Detection System, Host based IDSs, Network based IDSs, Misuse Detection, Anomaly Detection

\section{INTRODUCTION}

Intrusion Detection Systems (IDSs) are used to recognize and tale unauthorized or suspicious computer or network events. Host-based IDSs, the attention of this paper, are intended to monitor the host system actions, while networkbased IDSs observes network traffic for multiple hosts. Allowing to their detection techniques, IDSs can also be categorized into misuse detection or anomaly detection depending on whether the intrusion patterns are known or not throughout the design phase. Misuse detection approaches glance for predefined patterns or signatures related to accepted attacks, and therefore they are able to achieve a high level of detection accuracy. Though, misuse detection techniques cannot discover un-identified attacks for which signatures have not been detached yet (zero-day attacks) or well-known actions, which are able to variation their signatures with every implementation (polymorphic tacks) [1].

Normally, anomaly detection methods build profiles of expected normal behavior by means of training datasets that are composed over a period of normal system action. These datasets are collected in a protected environment, analyzed and clean to guarantee that the anomaly detector is trained on attack-free data. Throughout process, the anomaly detection system efforts to discover occasions that diverge meaningfully from the predictable normal profile. These deviations are cautions and specified as anomalous movements; though, they are not inescapably malicious doings as they may be shaped by software defects (e.g., coding or configuration errors) [5]. Anomaly detection procedures are talented of detecting novel attacks, though they are prone to make a large number of false alarms due mostly to the trouble in procurement a illustrative account of normal conduct of the system. The anomaly detectors will accordingly make an dangerous number of false alarms (by misclassifying rare normal events as anomalous), which could fail the trustworthiness of the anomaly detection system, mainly that the base-rate of normal minutes control the anomalous ones. Host-based anomaly discovery systems normally monitor for vital conflicts in operating system calls, as they offer a entry between user and kernel modes. Understandings presented that the historical order of system calls delivered by a process to request kernel services is real in effective normal process behavior [2]. This has entered to a large quantity of research that examined numerous methods for finding anomalies at the system call level. Amid these, order time-delay implanting (STIDE) and Hidden Markov Models (HMMs) are the most frequently used. Intrusion detection systems are mostly used calm with other defense systems such as approach control and validation as a second shield line to defend information systems. There are many details that make intrusion detection the key parts in the whole attack system. First, many of the old-style organisms and requests have been built and developed without taking safety extremely into account. Second, computer systems and applications may have errors or bugs in their plan that could be charity by burglars to attack the systems or applications. Hence, the preventive skill may not be as effective as anticipated [3].

\section{LITERATURE SURVEY}

Several unsupervised anomaly detection procedures have been useful to intrusion detection to improve IDSs recital in all levels such as in clustering, features selection and classifications. Erected on the prior illustration of the various unsupervised anomaly detection systems, Table 1 shows a evaluation among the most common processes. The contrast reviews the pros and cons of each one [6].

Relating machine learning skills for intrusion detection can repeatedly shape the model based on the training data set, which holds data instances that can be labelled by means of a usual of attributes (features) and associated labels. The attributes can be of countless sorts such as categorical or continuous [5].

The feebleness of knowledge base detection modus operandi. Anomaly detection comprehends supervised techniques and unsupervised techniques. Many procedures were used to realize good outcomes for these techniques. This paper suggests an impression of machine learning techniques for anomaly detection. The trials established that the supervised learning methods knowingly outstrip the unsupervised ones if the test data contains no unknown doses. Among the supervised ways and means, the best performance is completed by the non-linear methods, such as SVM, multi-layer perceptron and the rule-based means. 
Modus operandi for unsupervised such as K-Means, SOM, and one class SVM achieved better recital over the other skills although they differ in their competences of detecting all attacks classes proficiently [2].

\section{ANOAMALY DETECTION TECHNIQUES}

Relating machine learning skills for intrusion detection can repeatedly shape the model based on the training data set, which holds data instances that can be labelled by means of a usual of attributes (features) and associated labels. The attributes can be of countless sorts such as categorical or continuous [14].

Intrusion detection systems are mostly used calm with other defense systems such as approach control and validation as a second shield line to defend information systems. There are many details that make intrusion detection the key parts in the whole attack system. First, many of the old-style organisms and requests have been built and developed without taking safety extremely into account. Second, computer systems and applications may have errors or bugs in their plan that could be charity by burglars to attack the systems or applications. Hence, the preventive skill may not be as effective as anticipated [5].

\section{A. Nature of Input Data}

A crucial facet of any anomaly detection technique is the nature of the input data. Input is normally a collection of data instances. Each data instance can be described using a set of attributes. The attributes can be of altered types such as binary, categorical or continuous. To each data instance valor entail of only one attribute (univariate) or multiple attributes (multivariate) [6].

In the instance of multivariate data cases, all attributes capacity be of same type or might be a blend of different data types. Input data can also be categorized based on the relationship present among data instances.

Utmost of the existing anomaly detection techniques deal by record data (or point data), in which no relationship is implicit among the data instances [9].

\section{B. Type of Anomaly}

An important facet of an anomaly detection technique is the nature of the desired anomaly. Anomalies can be classified into following three categories:

1) Point Anomalies: If an distinct data instance can be careful as anomalous with respect to the rest of data, then the instance is dubbed as a point anomaly. This is the humblest type of anomaly and is the emphasis of majority of research on anomaly detection.

2)Contextual Anomalies: If a data instance in a exact context, then it is named as a contextual anomaly or conditional anomaly [7].

Intrusion Detection Systems (IDSs) are used to recognize and tale unauthorized or suspicious computer or network events. Host-based IDSs, the attention of this paper, are intended to monitor the host system actions, while networkbased IDSs observes network traffic for multiple hosts. Allowing to their detection techniques, IDSs can also be categorized into misuse detection or anomaly detection depending on whether the intrusion patterns are known or not throughout the design phase. Misuse detection approaches glance for predefined patterns or signatures related to accepted attacks, and therefore they are able to achieve a high level of detection accuracy. Though, misuse detection techniques cannot discover un-identified attacks for which signatures have not been detached yet (zero-day attacks) or well-known actions, which are able to variation their signatures with every implementation (polymorphic tacks) [14].

Normally, anomaly detection methods build profiles of expected normal behavior by means of training datasets that are composed over a period of normal system action. These datasets are collected in a protected environment, analyzed and clean to guarantee that the anomaly detector is trained on attack-free data. Throughout process, the anomaly detection system efforts to discover occasions that diverge meaningfully from the predictable normal profile. These deviations are cautions and specified as anomalous movements; though, they are not inescapably malicious doings as they may be shaped by software defects (e.g., coding or configuration errors) [11]. Anomaly detection procedures are talented of detecting novel attacks, though they are prone to make a large number of false alarms due mostly to the trouble in procurement a illustrative account of normal conduct of the system. The anomaly detectors will accordingly make an dangerous number of false alarms (by misclassifying rare normal events as anomalous), which could fail the trustworthiness of the anomaly detection system, mainly that the base-rate of normal minutes control the anomalous ones. Host-based anomaly discovery systems normally monitor for vital conflicts in operating system calls, as they offer a entry between user and kernel modes. Understandings presented that the historical order of system calls delivered by a process to request kernel services is real in effective normal process behavior [9]. This has entered to a large quantity of research that examined numerous methods for finding anomalies at the system call level. Amid these, order time-delay implanting (STIDE) and Hidden Markov Models (HMMs) are the most frequently used. Intrusion detection systems are mostly used calm with other defense systems such as approach control and validation as a second shield line to defend information systems. There are many details that make intrusion detection the key parts in the whole attack system. First, many of the old-style organisms and requests have been built and developed without taking safety extremely into account. Second, computer systems and applications may have errors or bugs in their plan that could be charity by burglars to attack the systems or applications. Hence, the preventive skill may not be as effective as anticipated [13].

The feebleness of knowledge base detection modus operandi. Anomaly detection comprehends supervised techniques and unsupervised techniques. Many procedures were used to realize good outcomes for these techniques. This paper suggests an impression of machine learning techniques for anomaly detection. The trials established that the supervised learning methods knowingly outstrip the unsupervised ones if the test data contains no unknown doses. Among the supervised ways and means, the best performance is completed by the non-linear methods, such as SVM, multi-layer perceptron and the rule-based means. Modus operandi for unsupervised such as K-Means, SOM, and one class SVM achieved better recital over the other skills although they differ in their competences of detecting all attacks classes proficiently [11].

Relating machine learning skills for intrusion detection can repeatedly shape the model based on the training data set, which holds data instances that can be labelled by means 


\begin{tabular}{|c|c|c|c|c|}
\hline $\begin{array}{c}\text { SI } \\
\text { No }\end{array}$ & Techniques & Abstract & Pros & Cons \\
\hline 1 & $\begin{array}{l}\text { K-Nearest } \\
\text { Neighbor }\end{array}$ & $\begin{array}{l}\text { K-nearest } \\
\text { neighbor is } \\
\text { one of the } \\
\text { unsure and } \\
\text { straight } \\
\text { modus } \\
\text { operandi for } \\
\text { classifying } \\
\text { samples. } \\
\text { It guesses the } \\
\text { rough } \\
\text { distances } \\
\text { amid several } \\
\text { points on the } \\
\text { input vectors, } \\
\text { and then allots } \\
\text { the unlabeled } \\
\text { point to the } \\
\text { class of its K- } \\
\text { nearest } \\
\text { neighbors. In } \\
\text { the course of } \\
\text { creating k-NN } \\
\text { classifier, (k) } \\
\text { is an key } \\
\text { parameter and } \\
\text { many } \\
\text { ethics can root } \\
\text { various } \\
\text { performances. } \\
\text { If k is very } \\
\text { vast, the } \\
\text { neighbors, } \\
\text { which charity } \\
\text { for prediction, } \\
\text { will consume } \\
\text { large } \\
\text { classification } \\
\text { time } \\
\text { move } \\
\text { prediction } \\
\text { accuracy [9]. }\end{array}$ & $\begin{array}{l}\text { - Very easy to } \\
\text { know when } \\
\text { there are rare } \\
\text { analyst } \\
\text { variables. } \\
\text { - Valuable for } \\
\text { building } \\
\text { replicas that } \\
\text { include non- } \\
\text { standard data } \\
\text { types, such as } \\
\text { text [12]. }\end{array}$ & $\begin{array}{l}\text { - Consume huge } \\
\text { storing } \\
\text { requirements. } \\
\text { - Sensitive to the } \\
\text { prime of the } \\
\text { similarity role } \\
\text { that is used to } \\
\text { liken instances. } \\
\text { - Absence a } \\
\text { upright means to } \\
\text { choose k, but } \\
\text { through cross- } \\
\text { validation or } \\
\text { similar. } \\
\text { - } \\
\text { Computationally } \\
\text { expensive } \\
\text { technique [8]. }\end{array}$ \\
\hline 2 & $\begin{array}{l}\text { Bayesian } \\
\text { Network }\end{array}$ & $\begin{array}{l}\text { Heckerman } \\
\text { defined a } \\
\text { Bayesian as } \\
\text { "A Bayesian } \\
\text { Network (BN) } \\
\text { is a model that } \\
\text { converts } \\
\text { probabilistic } \\
\text { relationships. } \\
\text { This } \\
\text { technique is } \\
\text { mostly used } \\
\text { for intrusion } \\
\text { detection in } \\
\text { mixture with } \\
\text { statistical } \\
\text { orders. It has } \\
\text { various the } \\
\text { advantages, } \\
\text { with the } \\
\text { capability of } \\
\text { encoding } \\
\text { amid variables } \\
\text { and of } \\
\text { predicting } \\
\text { actions, as } \\
\text { well as the } \\
\text { skill } \\
\text { incorporate } \\
\text { both prior } \\
\text { knowledge } \\
\text { and data [1]. }\end{array}$ & $\begin{array}{l}\text { - A neural } \\
\text { network can } \\
\text { do tasks that a } \\
\text { linear program } \\
\text { cannot. } \\
\text { - When an part } \\
\text { of the neural } \\
\text { network fails, } \\
\text { it can last } \\
\text { without any } \\
\text { tricky with } \\
\text { their parallel } \\
\text { nature. } \\
\text { - A neural } \\
\text { network crams } \\
\text { and does not } \\
\text { need to be } \\
\text { reprogrammed. } \\
\text { - It can be } \\
\text { applied in any } \\
\text { application } \\
\text { [14]. }\end{array}$ & $\begin{array}{l}\text { - The neural } \\
\text { network needs } \\
\text { training } \\
\text { operate. } \\
\text { - to } \\
\text { architecture of a } \\
\text { neural network } \\
\text { is different from } \\
\text { the architecture } \\
\text { of } \\
\text { microprocessors } \\
\text { therefore needs } \\
\text { to be emulated. } \\
\text { - Requires high } \\
\text { processing time } \\
\text { for large neural } \\
\text { networks [5]. }\end{array}$ \\
\hline
\end{tabular}

of a usual of attributes (features) and associated labels. The attributes can be of countless sorts such as categorical or continuous [10].

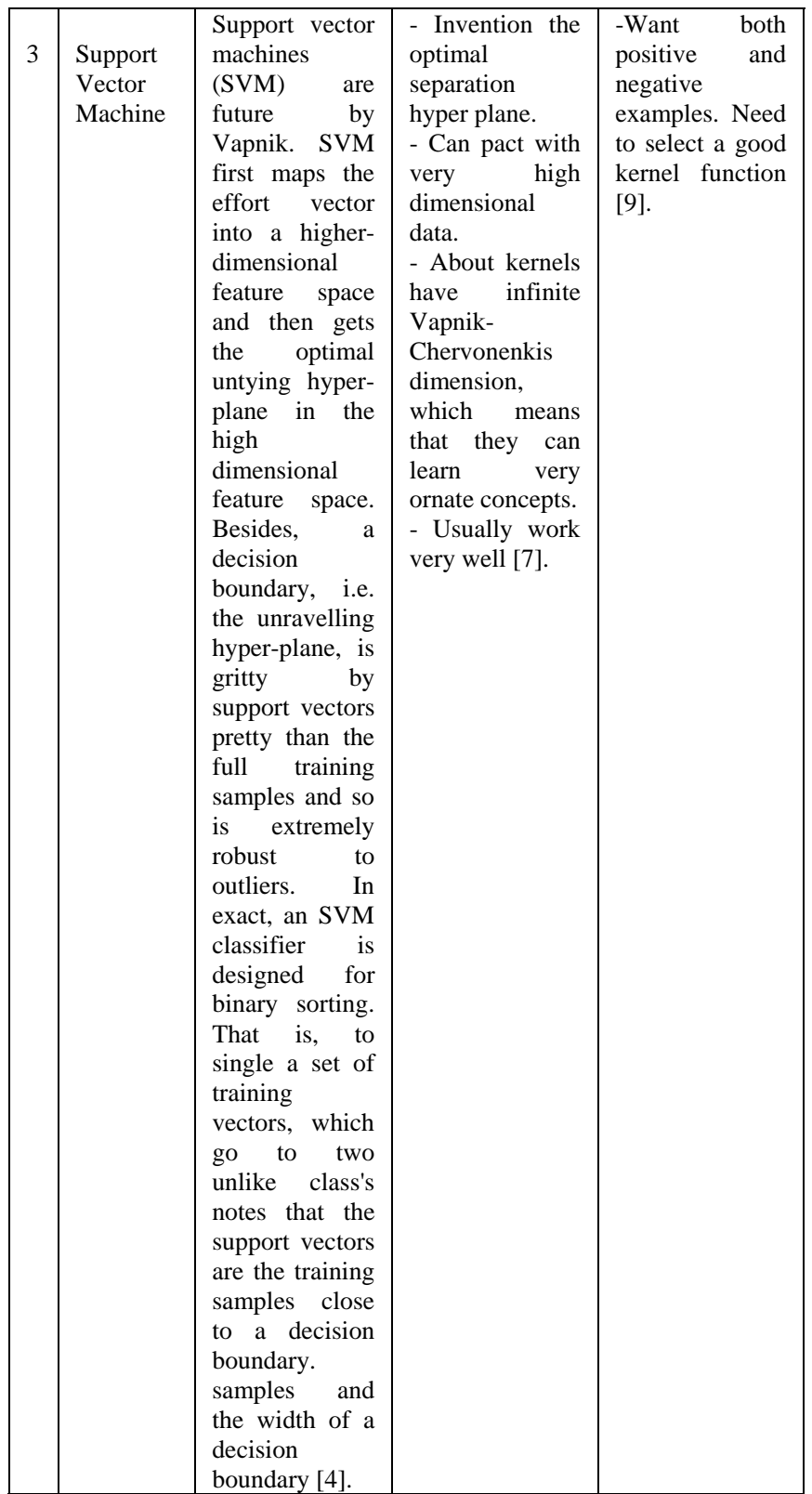

\section{REFERENCES}

[1] D.E. Denning, An intrusion detection model, in: Proceedings of the Seventh IEEE Symposium on Security and Privacy, 1986, pp. 119-131.

[2] J. McHugh, A. Christie, J. Allen, Defending yourself: the role of intrusion detection systems, IEEE Softw. 17 (5) (20 0 0) 42-51, doi: 10.1109/52.877859.

[3] S. Axelsson, Intrusion detection systems: a survey and taxonomy, in: Tech. Rep., Chalmers University, 2000, pp. 99-115.

[4] H.-J. Liao, C.-H.R. Lin, Y.-C. Lin, K.-Y. Tung, Intrusion detection system: a comprehensive review, J. Netw. Comput. Appl. 36 (1) (2013) 16-24, doi: 10.1016/j. jnca.2012.09.004.

[5] G.F. Cretu, A. Stavrou, M.E. Locasto, S.J. Stolfo, A.D. Keromytis, Casting out demons: sanitizing training data for anomaly sensors, in: IEEE Symposium on Security and Privacy, 2008. SP 2008., IEEE, 2008, pp. 81-95 . 
[6] C. Gates , C. Taylor, Challenging the anomaly detection paradigm: a provocative discussion, in: in: Proceedings of the 2006 Workshop on New Security Paradigms, NSPW '06, ACM, New York, NY, USA, 2006, pp. 21-29.

[7] R. Sommer, V. Paxson, Outside the closed world: On using machine learning for network intrusion detection, 2010. 0, 305-316.

[8] S. Forrest , S.A. Hofmeyr, A. Somayaji, T.A. Longstaff, A sense of self for Unix processes, in: Proceedings of the 1996 IEEE Symposium on Research in Security and Privacy, 1996, pp. 120-128 .

[9] Abdullah, B., Abd-algafar I., Salama G. I. and Abd-alhafez A. Performance Evaluation of a Genetic Algorithm Based Approach to Network Intrusion Detection System, Proceedings of 13th International Conference on Aerospace Sciences and Aviation Technology (ASAT-13), Military Technical College, Cairo, Egypt, 2009;1-5.
[10] Anderson, J. P. Computer security threat monitoring and surveillance. Technical Report, Fort Washington, PA, USA.,1980;9-11.

[11] Anderson, D., Frivold, T. and Valdes, A. Next-generation intrusion detection expert system (NIDES): A summary Technical Report SRI-CSL-95-07,Computer Science Laboratory,SRI International, May 1995.

[12] Beghdad, R. Critical study of neural networks in detecting intrusions. Computers and Security, 27(5-6): 2008;168175.

[13] Devikrishna, K. S. and Ramakrishna , B. B. .An Artificial Neural Network based Intrusion Detection System and Classification of Attacks", International Journal of Engineering Research and Applications (IJERA) ISSN: 2248-9622, Jul-Aug 2013, 3(4): 1959-1964.

[14] Denning, D. E.. An intrusion detection model, IEEE Transactions on Software Engineering, CA,. IEEE Computer Society Press;1987. 